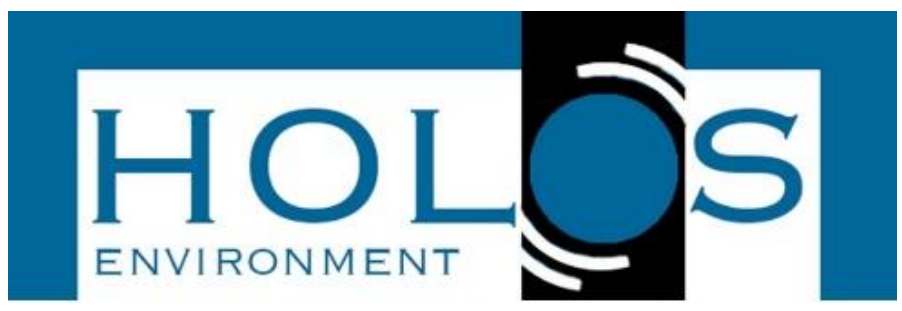

\title{
INVESTIGAÇÃO DO IMPACTO DO DESCARTE DE ÁGUA TERMAL NO RIO CAUIAZINHO, PRESIDENTE EPITÁCIO - SP
}

\section{INVESTIGATION OF THERMAL WATER DISPOSAL IMPACT IN THE CAIUAZINHO RIVER, PRESIDENTE EPITÁCIO - SP}

\author{
Elias Hideo Teramoto ${ }^{1}, 2$; Marcus Paulus Martins Baessa ${ }^{3}$; \\ Hung Kiang Chang ${ }^{1,2,4}$
}

Artigo recebido em: 19/12/2018 e aceito para publicação em: 06/05/2019.

DOI: http://dx.doi.org/10.14295/holos.v19i2.12324

Resumo: A água termal do Aquífero Guarani localizado no munícipio de Presidente Epitácio/SP, anteriormente utilizado para fins recreativos, é agora descartada para o Rio Caiuazinho, tributário do Rio Paraná. A temperatura da água medida na boca do poço é de $70^{\circ} \mathrm{C}$ e as características físico-químicas distintas daquelas observadas no Rio Caiuazinho. Diante das incertezas da influência do descarte de água quente na qualidade do rio, este trabalho pretendeu utilizar os isótopos estáveis e mensuração de parâmetros físico-químicos da água, a fim de se avaliar a influência da água termal no Aquífero Caiuá água subterrânea e superficial na área de interesse. Para alcançar os objetivos propostos foram coletadas 35 amostras de água superficial para análise isotópica, bem como realizada a medição de parâmetros físico-químicos em 48 pontos do Rio Caiuazinho em profundidades distintas, totalizando 224 medições. Os resultados isotópicos aliados à mensuração de parâmetros físico-químicos indicam que a vazão de água termal descartada, isto é, $34,5 \mathrm{~m}^{3} / \mathrm{h}$, não promovem alterações significativas nas características no rio.

Palavras-chave: Isótopos estáveis. SAG. Água termal.

Abstract: The thermal water of the Guarani Aquifer located in the municipality of Presidente Epitacio / SP, previously used for recreational purposes, is now ruled out for Cainazinho River, tributary of the Paraná River. The water temperature measured at the wellhead is $70^{\circ} \mathrm{C}$ and physicochemical characteristics different from those observed in Caiuazinho River. Considering the uncertainties of the influence of the hot water discharge quality of the river, this work aimed to use stable isotopes and measurement of water physical-chemical parameters, to evaluate the influence of thermal water in the Caiuá Aquifer and surface water in the area of interest. To achieve the proposed objectives were collected 32 samples of surface water for isotopic analysis and performed the measurement of physical-chemical parameters in 48 different points of Caiuazinho River at 3 different depths (totalizing 224 measurments). The isotopic results together with the measurement of physical and chemical parameters indicate that the flow of thermal water discarded, i.e. $34.5 \mathrm{~m}^{3} / \mathrm{h}$, not promote significant changes in the river's characteristics.

Palavras-chave: Stable isotopes. SAG. Thermal water.

\footnotetext{
${ }^{1}$ Lebac - Laboratório de Estudo de Bacias (Unesp). Campus de Rio Claro, SP. E-mails: (mailto:teramoto@rc.unesp.br, mailto:chang@rc.unesp.br)

2 CEA - Centro de Estudos Ambientais (Unesp), Campus de Rio Claro, SP.

${ }^{3}$ CENPES - Centro de Pesquisas Leopoldo Américo Miguez de Mello, Petrobras. E-mail: (marcus.baessa@petrobras.com.br)

4 DGA - Departamento de Geologia Aplicada (Unesp), Campus de Rio Claro, SP.
} 


\section{INTRODUÇÃO}

Os recursos hídricos em termos globais são afetados por uma mistura complexa de processos resultantes de fatores naturais ou induzidos por atividades antrópicas, incluindo o despejo de resíduos sem tratamento prévio, agricultura e ocupação urbana, geração de energia hidrelétrica e mudanças climáticas. Estes impactos se manifestam por alterações nas características físico-químicas originais da água, gerando estresse para os diferentes organismos aquáticos, uma vez que estas afetam as atividades fisiológicas e metabólicas destes organismos (HEUGENS et al., 2001). Dentre as alterações da água que são potencialmente nocivas às comunidades aquáticas, é possível destacar a temperatura, uma vez que este parâmetro controla as atividades fisiológicas destes organismos ectodérmicos, representando um fator crucial para a sobrevivência de destes orgânicos (HEUGENS et al., 2001; DADRAS et al., 2017; HORNSTEIN et al., 2018). A temperatura apresenta como um fator adverso adicional o incremento na toxicidade de metais dissolvidos na água (CAIRNS et al., 1975; HOLMSTRUP et al., 2010; BROWN et al., 2017).

Um poço estratigráfico, localizado no Município de Presidente Epitácio, Estado de São Paulo, foi perfurado pela Petrobras entre 1957 e 1959 para fins de prospecção de petróleo. A perfuração atingiu a profundidade final de 3953,5 metros e uma vez constatada a inexistência de potencial petrolífero na área, o poço foi adequadamente tamponado em 1959. Em 1987, o poço foi reaberto para produção de água termal proveniente do Sistema Aquífero Guarani (SAG), em profundidade de 1600-1700 m para ser empregado para fins de recreação em um parque de água termal. No ano de 2006, o DNPM (Departamento Nacional de Produção Mineral) interditou a fonte de água termal, lacrando a adução até o local denominado "Parque Thermas de Epitácio", devido à falta de autorização de lavra e pela água não estar em condições de consumo. Com o lacre das bombas pelo DNPM, a água termal passou a ser descartada diretamente no Rio Caiuazinho, tributário do Rio Paraná sem com os possíveis impactos deste descarte pudessem ser efetivamente compreendidos.

A proporção de efluente descartado em um corpo d'água superficial pode ser estimada por intermédio da análise isotópica da água. Esta estimativa é possível em razão da água de origens distintas possuírem diferentes combinações de moléculas ${ }^{1} \mathrm{H}_{2}{ }^{16}$, mais comuns com moléculas ${ }^{2} \mathrm{H}_{2}{ }^{16} \mathrm{O}$ e ${ }^{1} \mathrm{H}_{2}{ }^{18} \mathrm{O}$, mais raros. A composição isotópica da água é expressa em comparação com a composição isotópica média da água 
do oceano, um padrão internacional denominado Standard Mean Ocean Water (SMOW) e, atualmente, Viena Standard Mean Ocean Water (VSMOW) (CRAIG, 1961). A diferença entre a razão isotópica da água e este padrão é expressa em partes por mil (\%o). A razão de ${ }^{18} \mathrm{O}$ em relação ao ${ }^{16} \mathrm{O}$ é representada por $\delta^{18} \mathrm{O}$ e a de deutério em relação ao $\mathrm{H}$ é representada por $\delta^{2} \mathrm{H}$. Face à variabilidade natural observada na composição isotópica da água, a análise dos isótopos estáveis da água possibilita identificar a proveniência da água, bem como estimar a mistura destas águas. $\mathrm{O}$ emprego de isótopos estáveis para avaliar a porcentagem de mistura entre duas águas com composição isotópica distinta foi demonstrada em inúmeros trabalhos, incluindo CARTWRIGHT et al. (2012), CHEN et al. (2014), BINET et al. (2017).

O descarte de efluentes em corpos d'água superficiais pode promover alterações na composição e nos parâmetros físico-químicas da água, trazendo prejuízos ao metabolismo dos diversos orgânicos aquáticos. Diante da potencialidade existente nas técnicas isotópicas para estudo de recursos hídricos e das incertezas existentes quanto às interferências da água termal descartada indevidamente no Rio Caiuazinho, este trabalho pretendeu avaliar a magnitude de tais interferências, baseando-se nos parâmetros físico-químicos e na análise de isótopos estáveis da água deste rio.

\section{MATERIAIS E MÉTODOS}

\section{1 Área de Estudo}

O município de Presidente Epitácio se localiza no extremo noroeste do Estado de São Paulo às margens do Rio Paraná na divisa com o Estado de Mato Grosso do Sul, distando cerca de $645 \mathrm{Km}$ da cidade de São Paulo (Figura 1). A área de interesse situa-se a nordeste do perímetro urbano do munícipio de Presidente Epitácio, nas proximidades da desembocadura do Rio Caiuazinho com o Rio Paraná. A Figura 1 exibe a localização da área investigada, assim como a localização do poço de água termal e do local onde é destinada a água termal para descarte. 
Figura 1 - Localização do munícipio de Presidente Epitácio, onde se encontra a área estudada

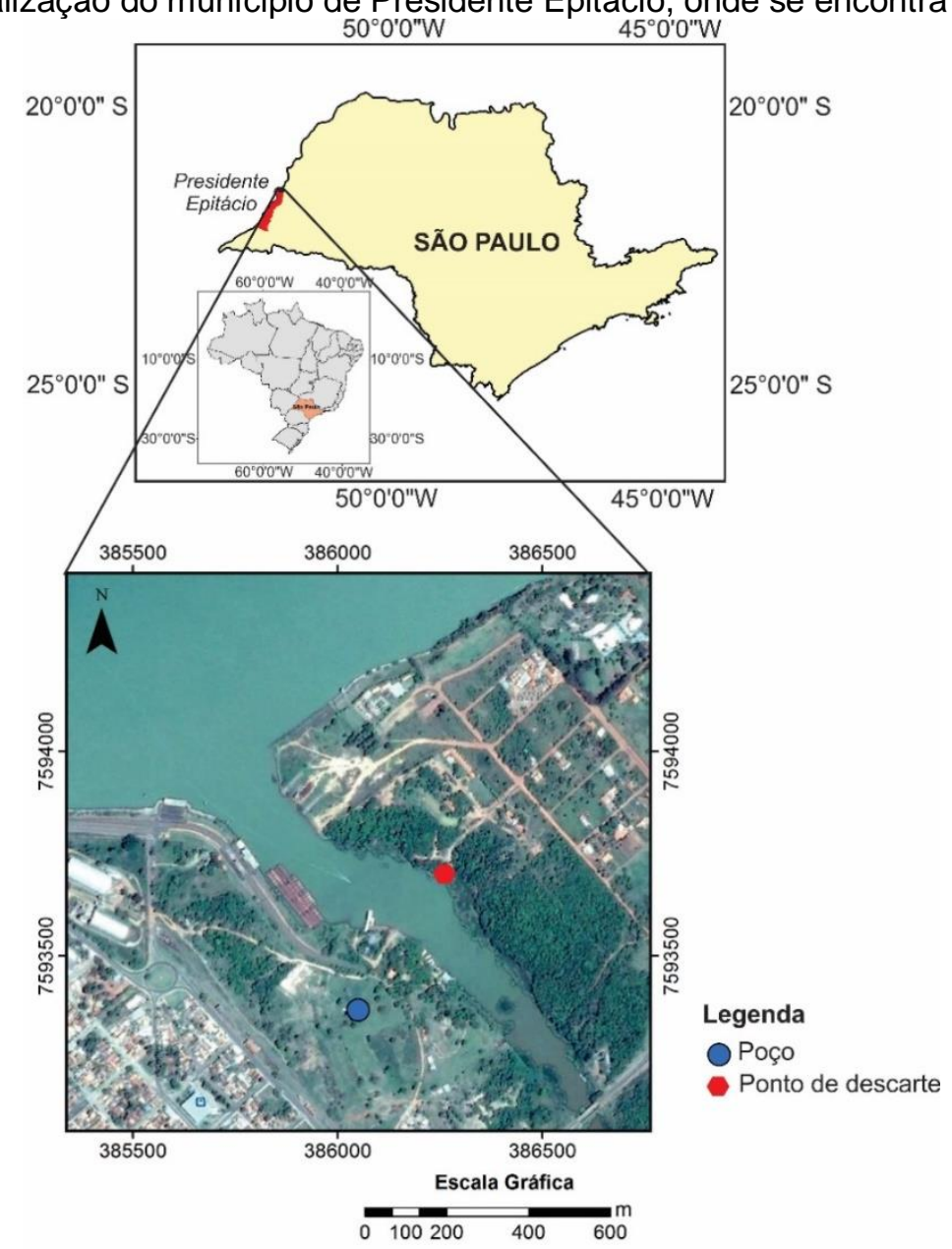

\subsection{Medição dos parâmetros físico-químicos}

A água do poço de água termal proveniente do Sistema Aquífero Guarani (SAG) possui composição química e características físico-químicas, OD (oxigênio dissolvidos), CE (condutividade elétrica da água), $\mathrm{pH}$ e temperatura, distintas daquelas observadas no Rio Caiuazinho. Em virtude desta diferença, é possível determinar a influência da água termal descartada no Rio Caiuazinho. Especial destaque foi destinado aos parâmetros temperatura e CE por representarem os parâmetros que exibem maior contraste entre as águas do SAG e do Rio Caiuazinho.

Com o emprego da sonda multi-parâmetros da marca Yellow Spring com 10 metros de cabo foram realizadas medições dos parâmetros temperatura e condutividade elétrica da água em 48 pontos do ao longo do Rio Caiuazinho. Em cada um dos 48 pontos foi promovida a medição em três intervalos de profundidades distintos, sendo a primeira medida na superfície do rio, a segunda à 1 metro e a terceira 
à 2 metros de profundidade, totalizando 124 medições. As medições foram registradas somente após a estabilização dos parâmetros lidos, de modo a se assegurar a validade dos valores registrados e o posicionamento geográfico de cada amostra foi determinado com o auxílio de GPS.

Figura 2 - Localização das amostras de água superficial para análise isotópica.

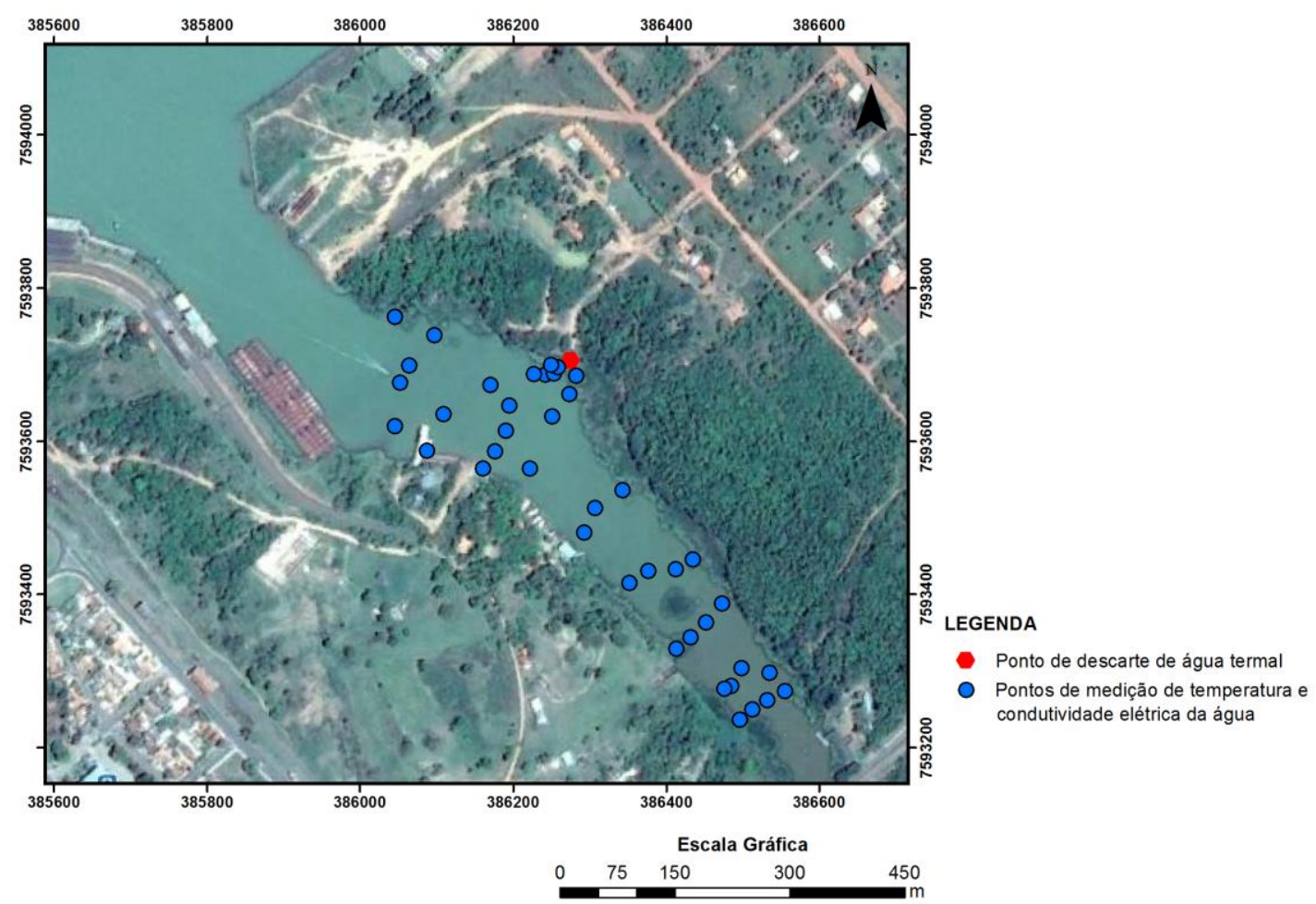

\subsection{Amostragem de água para análise isotópica}

Concomitante à realização dos parâmetros físico-químicos, foi conduzida a coleta de amostras de 32 amostras de água do Rio Caiuazinho para análise da composição isotópica. As análises isotópicas pretenderam caracterizar a existência de assinaturas isotópicas naturais ou decorrentes da mistura com água termal, foram amostradas de água em diferentes intervalos de profundidade distintas. Imediatamente após a coleta, os frascos foram fechados e conservados sob refrigeração até sua destinação, no laboratório de análises isotópicas.

Para quantificar o impacto do despejo de água termal no Rio Caiuazinho, foi realizada a coleta de uma amostra do poço de água termal do aquífero Guarani para fins de análise isotópica. De posse dos valores das assinaturas da água natural do Rio Caiuazinho e da água termal foi estimada as proporções de mistura entre estas 
duas águas no Rio Caiuazinho em amostras que se alinhavam graficamente nesta linha de mistura, a partir do procedimento descrito por CLARK \& FRITZ (2004).

\subsection{Procedimento analítico para análise isotópica}

As análises dos isótopos estáveis de oxigênio e hidrogênio foram realizadas por espectroscopia isotópica de absorção laser em cavidade ressonante. Essa técnica, do tipo ringdown (CRDS, cavity ring-down spectroscopy), se baseia na absorção de radiação laser na faixa do infravermelho próximo (0,7 mícrons a 2,5 mícrons) por substâncias no estado gasoso, como água, gás carbônico e metano. $O$ instrumento utilizado para análise isotópica foi Liquid Water Isotope Analyzer da Los Gatos Research Inc (LWIA-24d). As análises isotópicas $\left(\delta^{18} \mathrm{O}\right.$ e de $\left.\delta^{2} \mathrm{H}\right)$ foram efetuadas no laboratório de Hidroquímica do IGCE, UNESP, Rio Claro.

Como exposto por CLARK \& FRITZ (2007), os isótopos de ${ }^{18} \mathrm{O}$ e ${ }^{2} \mathrm{H}$ são conservativos em processos de misturas de águas com origens distintas. Isto é, em uma mistura não haverá incremento ou perda destes isótopos e a assinatura isotópica será linearmente dependente da fração de cada fonte na mistura. Neste contexto, as diferentes proporções desta mistura se alinham entre duas fontes originais de água no gráfico de $\delta^{2} \mathrm{H}$ versus $\delta^{18} \mathrm{O}$. Adotando essa premissa como verdadeira, a proporção de mistura da água termal do SAG no Rio Caiuazinho foi estimada graficamente a partir dos resultados de análise isotópica.

\section{RESULTADOS}

\subsection{Análises físico-químicas}

\subsubsection{Parâmetros físico-químicos da água termal}

A água termal do SAG foi coletada diretamente na boca do poço, do tipo jorrante. A temperatura da água era de $70^{\circ} \mathrm{C}$, a condutividade de $1187 \mathrm{mS} / \mathrm{cm}$ e o pH de 9,2 . No ponto de descarte, os parâmetros físico-químicos se mantiveram similares, mas a temperatura da água medida nesse ponto foi de $61^{\circ} \mathrm{C}$. 
3.1.2 Avaliação de alterações nos parâmetros físico-químicos do Rio Caiuazinho

Uma vez que os parâmetros físico-químicos do Rio Caiuazinho e da água termal do SAG são bastante constantes, a influência da água termal pode ser identificada por alterações nestes parâmetros no rio. A Tabela 1 apresenta a comparação dos parâmetros físico-químicos da água termal do $S A G$ e da amostra de água do Rio Caiuazinho medido no ponto mais distante do ponto de descarte.

\begin{tabular}{|c|c|c|c|c|}
\hline Amostra & $\mathbf{T}\left({ }^{\circ} \mathrm{C}\right)$ & CE $(\mu \mathrm{S} / \mathrm{cm})$ & $O D(\mathrm{mg} / \mathrm{L})$ & $\mathrm{pH}$ \\
\hline $1 \mathrm{D}$ & 26,4 & 84,1 & 4,29 & 6,47 \\
\hline Água termal & 61,0 & 1187 & 3,77 & 8,79 \\
\hline
\end{tabular}

O valor de condutividade elétrica da água termal é de $1187 \mu \mathrm{S} / \mathrm{cm}$, superior àquelas observadas no Rio Caiuazinho e decorrentes das elevadas concentrações de $\mathrm{HCO}_{3}{ }^{-}$e o $\mathrm{Cl}^{-} . \mathrm{O} \mathrm{pH}$ elevado reflete provavelmente o equilíbrio com o mineral calcita $\left(\mathrm{CaCO}_{3}\right)$ em um sistema fechado em relação ao $\mathrm{CO}_{2}$. Por outro lado, a água do rio à montante do ponto de descarte varia entre 69,5 e $88,5 \mathrm{mS} / \mathrm{cm}$, pH próximo da neutralidade em razão do equilíbrio com o $\mathrm{CO}_{2}$ atmosférico e baixas concentrações de carbonatos.

Em razão do contraste existente entre os dois membros finais, a mensuração dos diferentes parâmetros físico-químicos permite avaliar preliminarmente a influência da água termal no corpo d'água superficial. A Figura 3 apresenta os histogramas dos parâmetros físico-químicos medidos nas 124 medições realizadas no Rio Caiuazinho (Figura 2). 
Figura 3 - Histogramas dos parâmetros físico-químicos medidos no Rio Caiuazinho: a) Tem peratura; b) Oxigênio dissolvido;

c) Condutividade Elétrica; d) $\mathrm{pH}$.
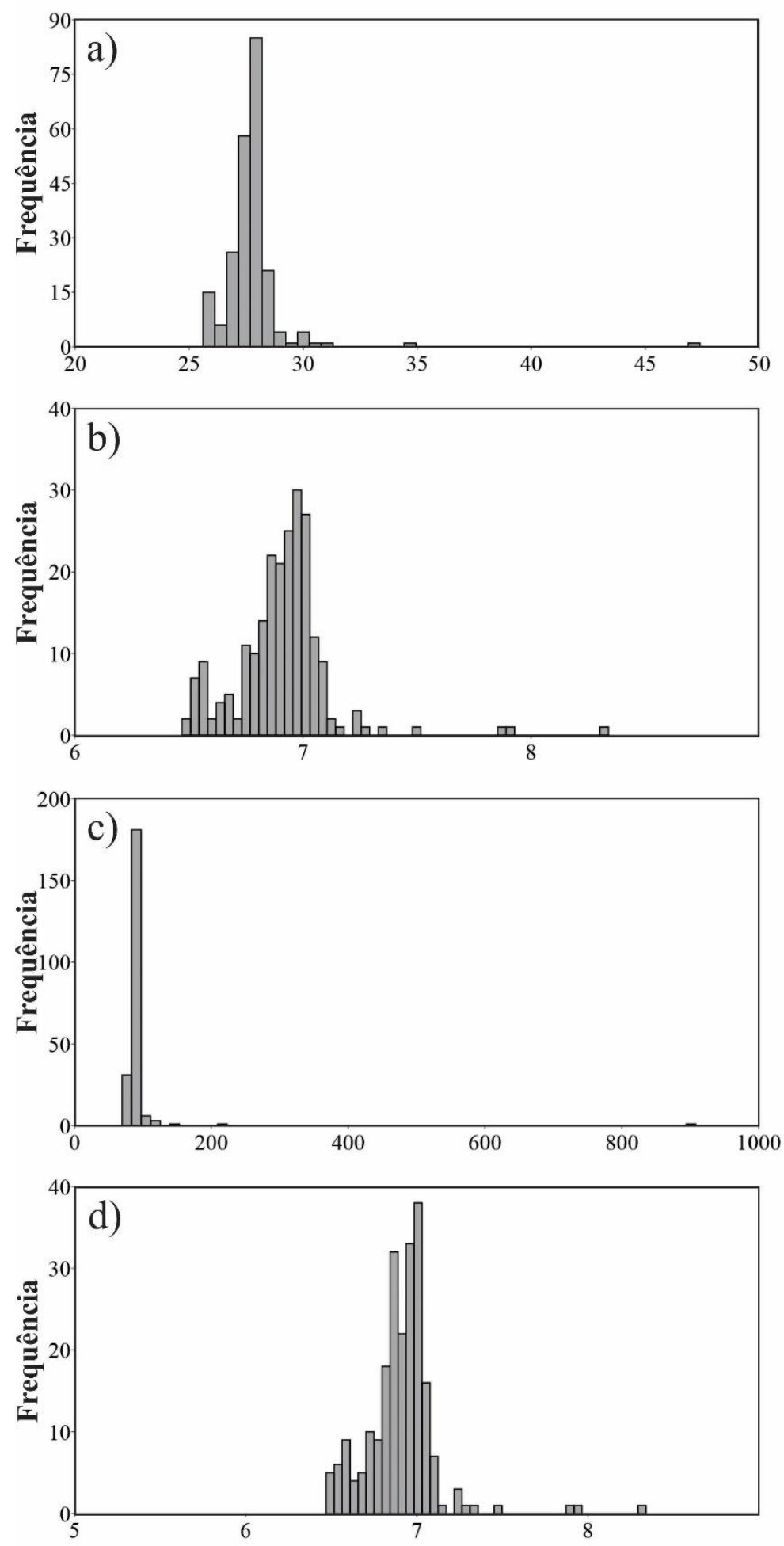

\subsubsection{Temperatura do Rio Caiuazinho}

A temperatura da água representou o principal parâmetro de interesse no presente estudo, tendo em vista que o descarte de água termal pode incrementar 
significativamente a temperatura da água do rio, podendo causar danos à biota aquática. A Figura 4a ilustra a temperatura da água na superfície do rio, onde é possível observar a existência de um gradiente, onde as menores temperaturas estão na porção sudeste, a montante, e tornam-se mais quentes na direção noroeste a jusante do fluxo. As Figuras $3 \mathrm{~b}$ e $3 \mathrm{c}$ ilustram respectivamente as temperaturas medidas a $1 \mathrm{me} 2 \mathrm{~m}$ de profundidade e indicam que as temperaturas a elevação da temperatura são significativas na superfície, mas pouco aparente em porções mais profundas.

Figura 4 - Mapa de isovalores de temperatura da água mensurada no Rio Caiuazinho para: a) superfície do rio; b) 1,0 m de profundidade; c) 2,0 $\mathrm{m}$ de profundidade.
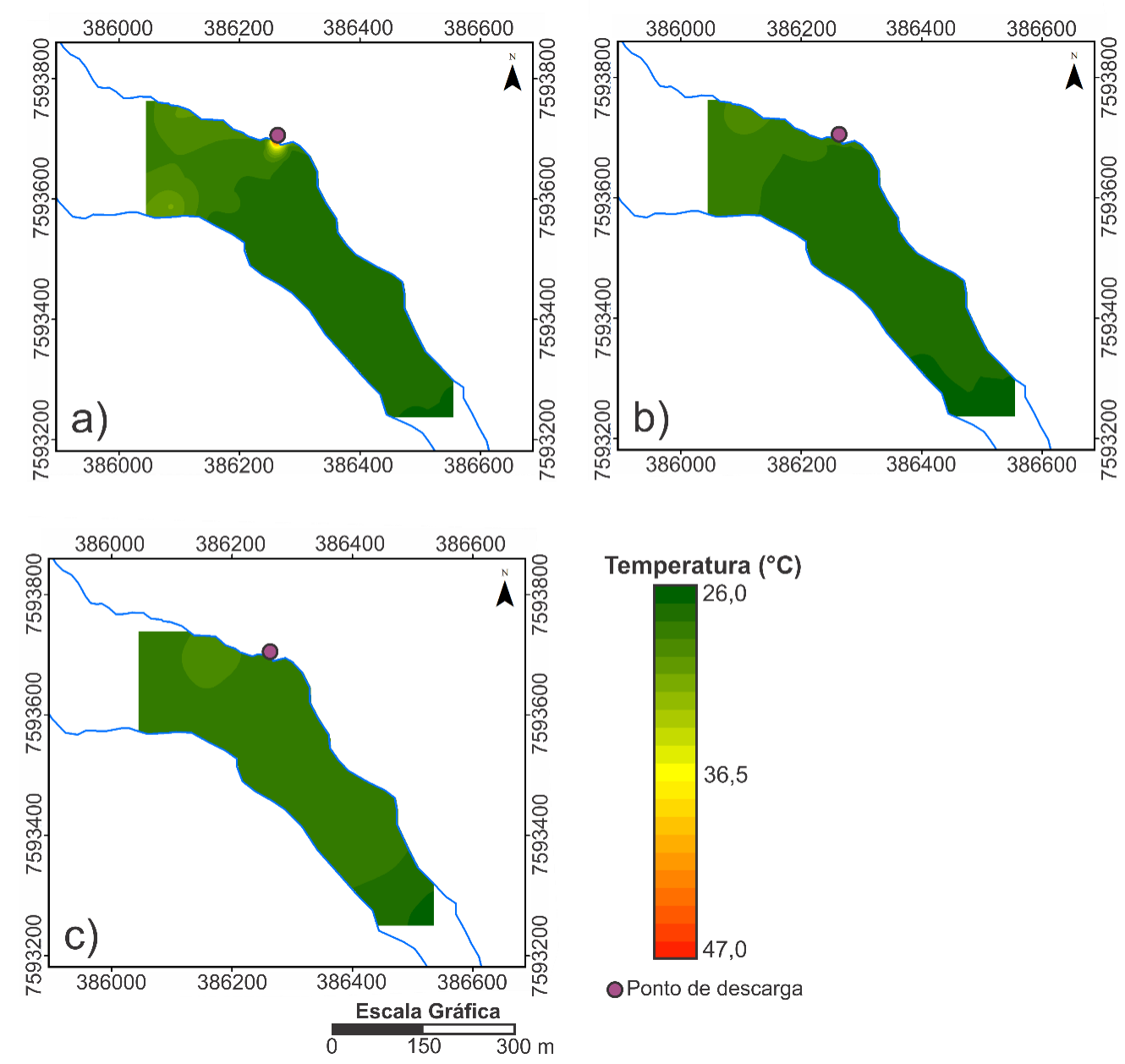

Para avaliar a dependência das variações dos parâmetros $\mathrm{CE}, \mathrm{pH}$ e OD em função da mistura com a água termal, foram construídos gráficos de dispersão entre estes parâmetros e a temperatura da água (Figura 5). As Figuras $5 a$ e $5 b$ indicam que valores de $\mathrm{CE}$ e pH possuem correlação elevada (Figura 5a) ou moderada (Figura 5b) com a temperatura da água, sugerindo que a variação destes parâmetros se dá pela 
mistura com água termal. Entretanto, é possível notar que a presença de valores extremos de temperatura, $\mathrm{CE}$ e $\mathrm{pH}$ controlam os valores de $\mathrm{R}^{2}$ determinados. A Figura $5 c$, por outro lado, indica que a inexistência de correlação entre o parâmetro OD e a temperatura da água, uma vez que o coeficiente de determinação $\left(R^{2}\right)$ é igual a 0,0904 , indicando que a possível mistura da água termal não interfere na quantidade de oxigênio dissolvido no rio.

Figura 5 - Gráficos de dispersão entre temperatura e demais parâmetros físico-químicos: a)
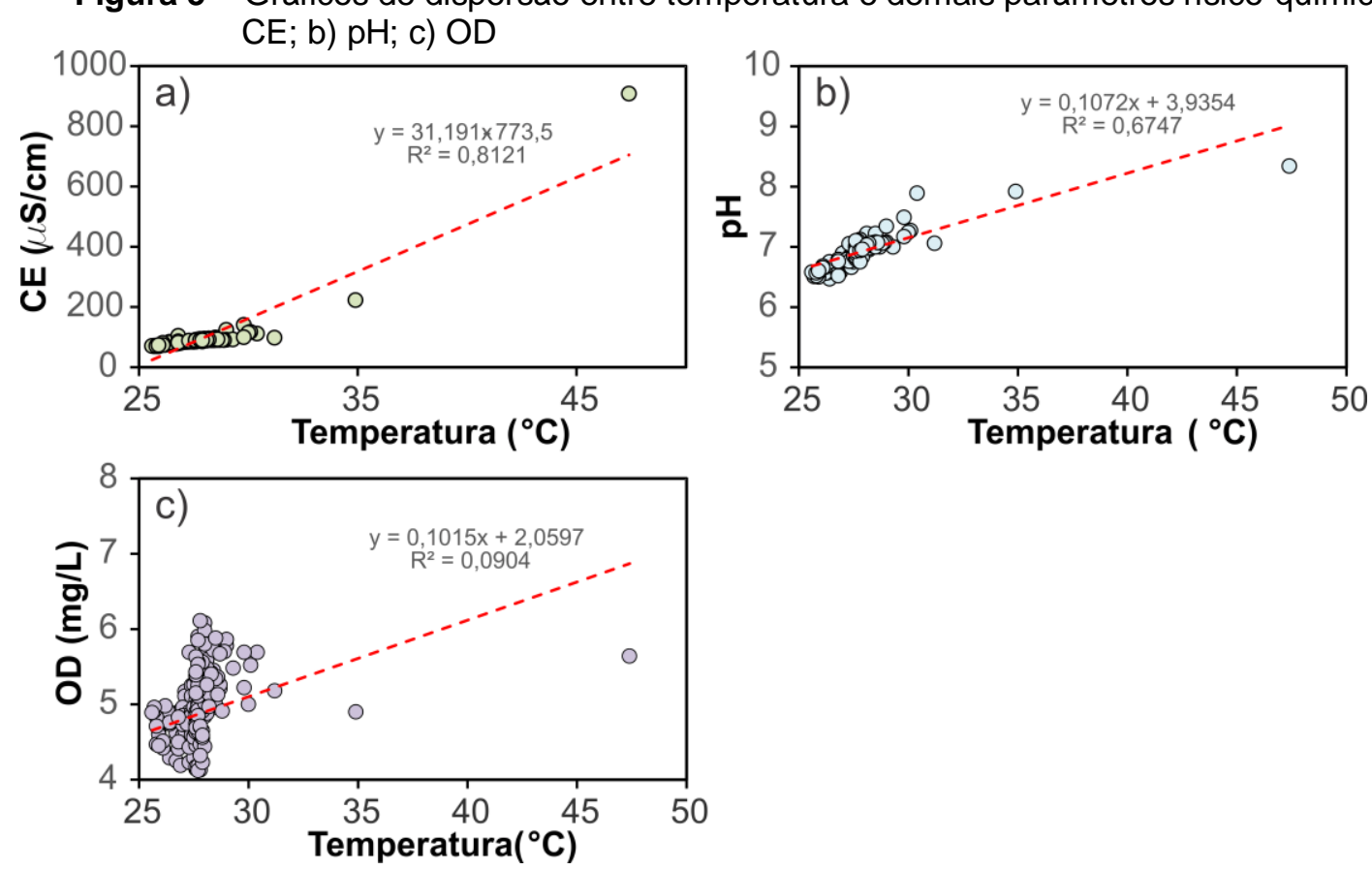

\subsection{Análise isotópica}

\subsection{1Água termal do SAG}

A assinatura isotópica da água termal do SAG coletada no ponto de descarte foi $\delta^{2} \mathrm{H}$ igual a $-47,7$ e $\delta^{18} \mathrm{O}$ igual a $-6,72$.

\subsection{2Água superficial}

A Tabela 2 apresenta os resultados isotópicos de todas as amostras coletadas no Rio Caiuazinho, onde é possível observar uma grande variabilidade nos isótopos de interesse. 
Tabela 2 - Localização e resultados das análises isotópicas de amostras de água coletadas no Rio Caiuazinho

\begin{tabular}{cccccc}
\hline ID & X UTM $(\mathbf{m})$ & Y UTM $(\mathbf{m})$ & Profundidade $(\mathbf{m})$ & $\boldsymbol{\delta}^{2} \mathbf{H}(\% \mathbf{0})$ & $\mathbf{\delta}^{18} \mathbf{O}(\% \mathbf{)})$ \\
\hline P1 & 389522 & 7591721 & 0,0 & $-37,9$ & $-6,19$ \\
P2 & 386765 & 7593082 & 0,0 & $-37,7$ & $-6,03$ \\
P3 & 386512 & 7593250 & 0,5 & $-32,0$ & $-5,35$ \\
P4A & 386532 & 7593262 & 0,0 & $-31,9$ & $-5,30$ \\
P4B & 386532 & 7593262 & 0,5 & $-33,9$ & $-5,42$ \\
P5 & 386555 & 7593274 & 0,5 & $-32,0$ & $-5,27$ \\
P6A & 386535 & 7593298 & 0,0 & $-31,9$ & $-5,06$ \\
P6B & 386535 & 7593298 & 0,5 & $-34,1$ & $-5,18$ \\
P7A & 386498 & 7593304 & 0,0 & $-32,6$ & $-5,19$ \\
P7B & 386498 & 7593304 & 0,5 & $-36,5$ & $-5,93$ \\
P8 A & 386485 & 7593281 & 0,0 & $-32,0$ & $-5,14$ \\
P9 & 386476 & 7593277 & 0,0 & $-32,6$ & $-5,14$ \\
P10 & 386452 & 7593364 & 3,0 & $-36,4$ & $-5,96$ \\
P11 & 386352 & 7593415 & 0,0 & $-32,0$ & $-5,76$ \\
P12 & 386343 & 7593536 & 0,0 & $-32,6$ & $-5,66$ \\
P13 & 386282 & 7593686 & 0,0 & $-31,4$ & $-5,15$ \\
P14 & 386273 & 7593662 & 0,0 & $-31,9$ & $-5,21$ \\
P15 & 386251 & 7593633 & 0,0 & $-32,0$ & $-5,18$ \\
P16 & 386222 & 7593565 & 0,0 & $-31,5$ & $-5,00$ \\
P17 & 386190 & 7593614 & 0,0 & $-31,6$ & $-4,93$ \\
P18 & 386195 & 7593647 & 0,0 & $-31,7$ & $-4,85$ \\
P19 & 386242 & 7593687 & 0,0 & $-32,2$ & $-4,72$ \\
P20A & 386253 & 7593689 & 0,0 & $-39,1$ & $-5,58$ \\
P20B & 386253 & 7593689 & 1,0 & $-32,1$ & $-4,71$ \\
P21 & 386258 & 7593697 & 0,0 & $-45,9$ & $-6,27$ \\
P22A & 386249 & 7593700 & 0,0 & $-36,8$ & $-5,28$ \\
P22B & 386249 & 7593700 & 0,5 & $-31,6$ & $-5,22$ \\
P23 & 386227 & 7593688 & 0,0 & $-32,3$ & $-5,06$ \\
P24 & 386170 & 7593674 & 0,0 & $-32,0$ & $-5,05$ \\
P25 & 386109 & 7593636 & 0,0 & $-32,0$ & $-5,21$ \\
P26 & 386052 & 7593677 & 0,0 & $-31,2$ & $-5,06$ \\
P27 & 386097 & 7593739 & 0,0 & $-32,0$ & $-5,13$ \\
P28 & 386045 & 7593763 & 0,0 & $-31,4$ & $-4,91$ \\
\hline & & & & &
\end{tabular}

A Figura 6 apresenta o gráfico de dispersão dos valores de $\delta^{2} \mathrm{H}$ em função dos valores de $\delta^{18} \mathrm{O}$, bem como a amostra de água termal do SAG e o cálculo de mistura nas amostras nas quais esta mistura é evidente. 
Figura 6 - Gráfico de dispersão de ${ }^{2} \mathrm{H}$ e ${ }^{18} \mathrm{O}$ do conjunto de amostras de águas superficiais e subterrâneas coletadas e cálculo da mistura de água termal nas águas superficiais do Rio Caiuazinho.

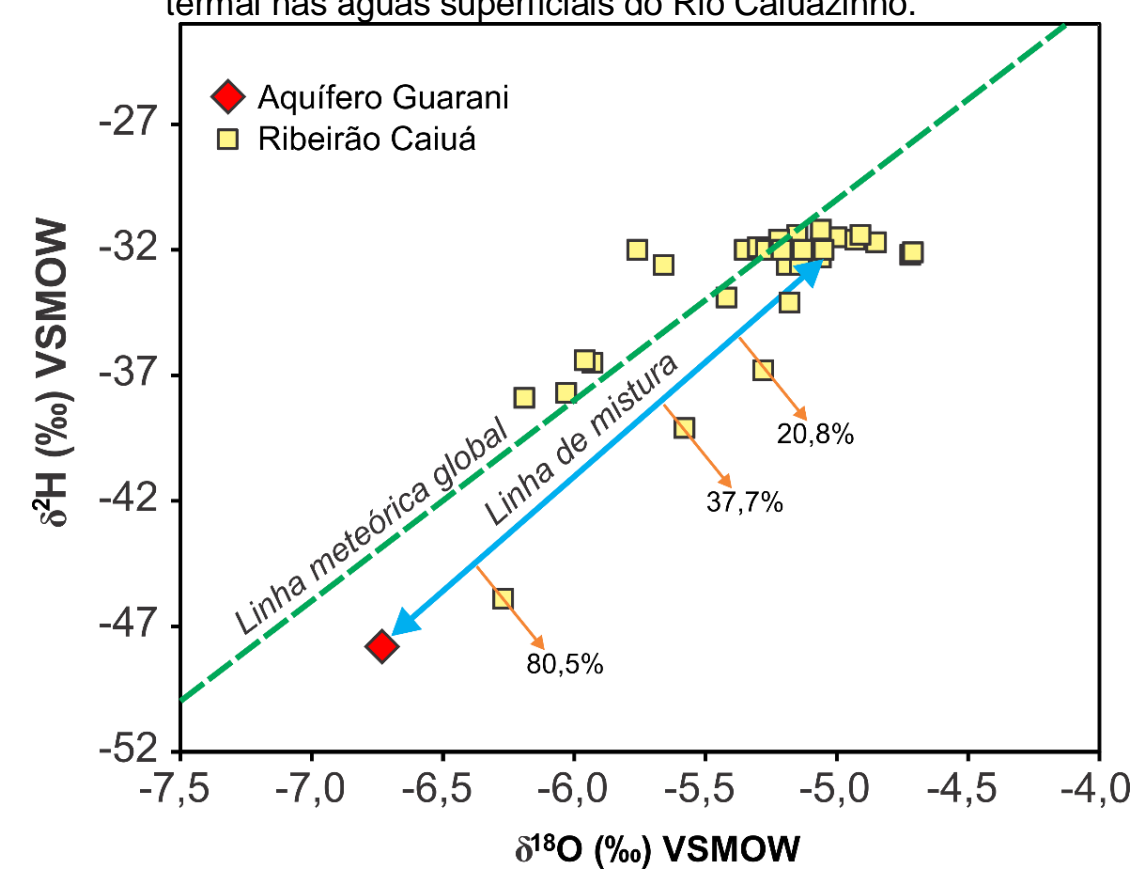

É possível notar que apenas 3 das 28 amostras coletadas apresentam evidências de mistura com a água termal.

Tendo em vista que a temperatura e a análise isotópica da água do Rio Caiuazinho são resultantes da mistura com a água termal, foi construído um gráfico de dispersão entre a temperatura e os valores de $\delta^{2} \mathrm{H}$ (Figura 7).

Figura 7 - Gráfico de dispersão entre temperatura e $\delta^{2} \mathrm{H}$, demonstrando a existência de uma correlação negativa entre estes parâmetros

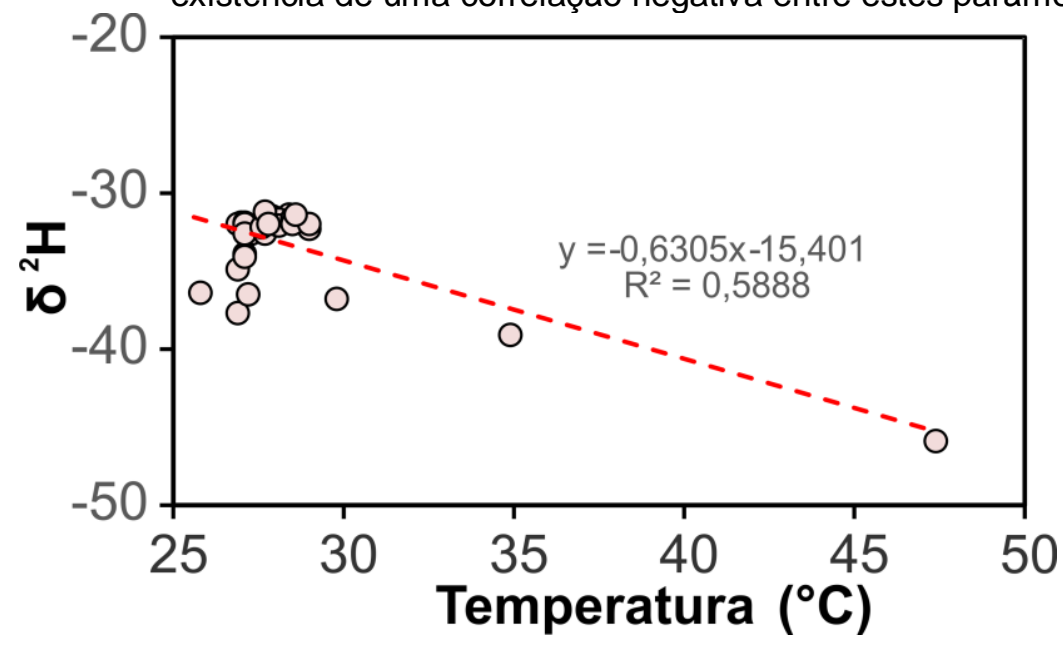




\section{DISCUSSÕES}

Duas abordagens distintas foram empregadas para investigar a influência da água termal no rio. A primeira abordagem consistiu na medição dos parâmetros físicoquímicos da água do rio para identificar a presença de anomalias em tais parâmetros e a extensão destas. A segunda abordagem consistiu em técnicas de análise isotópica da água para determinar quantitativamente a porcentagem de mistura de água termal no rio.

Em razão do elevado contraste nos parâmetros físico-químicos do SAG e do Rio Caiuazinho, o descarte de água termal resultaria em modificações perceptíveis das características originais do rio. Sob a hipótese a progressiva mistura entre estas duas águas resultasse em águas com características intermediárias entre ambas, era esperado uma ampla dispersão dos valores medidos e um histograma largo, com desvio-padrão elevado. No entanto, os histogramas dos parâmetros temperatura (Figura 3a), oxigênio dissolvido (Figura 3b), condutividade elétrica (Figura 3c) e pH (Figura 3d), indicam baixa variabilidade dos valores medidos destes parâmetros. Tendo em vista que os valores dos parâmetros físico-químicos medidos estão distantes daqueles observados na água termal descartada, as variações observadas nestes parâmetros são na maior parte resultantes de variações naturais no rio. Entretanto, é possível notar a presença outliers nos histogramas, que podem ser atribuídos à influência da água termal, uma vez que tais amostras foram coletadas mais próximas do ponto de descarte.

Os mapas de temperatura do Rio Caiuazinho (Figura 4) indicam a existência de uma tênue elevação na temperatura da água, sendo possível observar que existe uma tendência da temperatura medida à jusante do ponto de descarte é superior à temperatura medida à montante. É possível notar que as anomalias de temperatura estão restritas às proximidades do ponto de descarte de água termal. A temperatura medida no ponto de descarte para o rio é de $61^{\circ} \mathrm{C}$ (cerca de $10^{\circ} \mathrm{C}$ inferior à temperatura medida no poço), enquanto a temperatura máxima medida no rio foi de $47^{\circ} \mathrm{C}$ a 1 metro do ponto de descarte, caindo para $34{ }^{\circ} \mathrm{C}$ a 5 metros do ponto de descarga (Figura 4), sendo este fato atribuído à tendência da rápida dissipação da temperatura em virtude da mistura com a água do rio. A comparação da Figura 4a com as Figuras $4 \mathrm{~b}$ e $4 \mathrm{c}$ indica que as temperaturas anômalas ocorrem somente na superfície do rio e 
porções mais profundas do rio não são diretamente afetadas pela elevação de temperatura.

Visando determinar a proporção da mistura de água termal no Rio Caiuazinho, foram coletadas 28 amostras de água para análise isotópica. A determinação da proporção de mistura é possível, uma vez que identificou assinaturas isotópicas distintas entre a água termal descartada e a água do rio. A Figura 5 indica que o parâmetro CE apresenta boa correlação com a temperatura, enquanto o parâmetro pH apresenta apenas uma correlação moderada. Entretanto, os valores de coeficiente de terminação calculados são fortemente influenciados pela presença de valores anomalamente elevados de temperatura, $\mathrm{CE}$ e pH e atribuídos à influência de água termal. É provável que a remoção destes valores elevados da análise promova uma redução substancial dos valores do coeficiente de determinação $\left(R^{2}\right)$ entre estes parâmetros e a temperatura. A ausência de correlação entre a temperatura e o OD indica que o descarte de água termal não se manifesta a partir da redução da quantidade de oxigênio na água, ao contrário do que teorizado a priori, tendo em vista que a solubilidade do oxigênio diminui com a temperatura.

Na Figura 6 é possível importante notar que parte das amostras de água do Rio Caiuazinho se situa próximo da linha meteórica global, indicando pouca influência do enriquecimento isotópico decorrente da evaporação e parte apresenta um deslocamento negativo em relação à linha meteórica global, indicando a influência da evaporação. O efeito da evaporação é especialmente significado nas proximidades da desembocadura do Rio Paraná, onde o Rio Caiuazinho se alarga, apresentando uma ampla área superficial sujeita à irradiação solar. Como observado na Tabela 1, as amostras de água de porções mais rasas do Rio Caiuazinho são mais pesadas que as amostras de água coletadas em porções mais profundas, indicando que o efeito da evaporação é mais intenso na porção superior do rio e menos pronunciado em porções mais profundas, gerando uma estratificação isotópica no rio.

A Figura 7 demonstra uma correlação negativa entre a temperatura e $\circ \delta^{2} \mathrm{H}$, indicando que a assinatura isotópica e a temperatura consistentemente são indicativas da mistura com a água termal. Tendo em vista que a água termal possui valores de $\delta^{2} \mathrm{H}$ mais negativos, o incremento de proporção de mistura com a água termal torna a água mais negativa em relação ao deutério. De maneira similar à Figura 6, a Figura 7 indica que apenas 3 amostras apresentam influência da mistura com a água termal. 
Do conjunto de 28 amostras coletadas, apenas três (P20A, P21 e P22A), coletadas nas proximidades do ponto de descarte de água termal, apresentaram indícios de mistura com a água do SAG e estão de acordo com as medições dos parâmetros físico-químicos medidos. A proporção de mistura nestas amostras pode ser calculada graficamente a partir da determinação da distância de cada ponto de mistura em relação ao ponto que representa a amostra de água termal, dividido pela distância entre a água termal e a água do rio. Adotando esse procedimento, obteve-se valores de $80,5 \%, 37,7 \%$ e $20,6 \%$ de água termal na mistura nas 3 amostras que apresentaram indícios de mistura. Tal comportamento é consistente com os valores de temperatura medidos, apresentados nas Figuras 4a-c, que indicam que modificações na temperatura são significativas somente nas proximidades do ponto de descarte de água termal. As duas abordagens empregadas no presente trabalho fornecem os mesmos resultados, apontando para interferências pouco significativas da água termal descartada no Rio Caiuazinho.

\section{CONCLUSÕES}

A medição dos parâmetros físico-químicos em 224 pontos distintos do Rio Caiuazinho indica que o descarte de água termal não promove alterações significativas nas características originais deste rio. Variações significativas nos parâmetros físico-químicos e, particularmente na temperatura, são observadas apenas nas proximidades do ponto de descarte. Esta constatação é reforçada pela caracterização isotópica em amostras de água coletadas no rio, que indicam que apenas 3 das 28 amostras analisadas apresentam algum tipo de mistura de água termal. Estes resultados sugerem que a água termal do SAG descartada no Rio Caiuazinho é rapidamente diluída e o seu impacto é bastante limitado.

\section{REFERÊNCIAS}

BINET, Stéphane et al. Water exchange, mixing and transient storage between a saturated karstic conduit and the surrounding aquifer: groundwater flow modeling and inputs from stable water isotopes. Journal of Hydrology, v. 544, p. 278-289, 2017.

BROWN, Alastair; THATJE, Sven; HAUTON, Chris. The effects of temperature and hydrostatic pressure on metal toxicity: insights into toxicity in the deep sea. Environmental science \& technology, v. 51, n. 17, p. 10222-10231, 2017. 
CAIRNS, John; HEATH, Alan G.; PARKER, Bruce C. The effects of temperature upon the toxicity of chemicals to aquatic organisms. Hydrobiologia, v. 47, n. 1, p. 135-171, 1975.

CARTWRIGHT, lan et al. Constraining groundwater flow, residence times, inter-aquifer mixing, and aquifer properties using environmental isotopes in the southeast Murray Basin, Australia. Applied geochemistry, v. 27, n. 9, p. 1698-1709, 2012.

CLARK, Ian D.; FRITZ, Peter. Environmental Isotopes in Hydrogeology. CRC press, 2013.

CRAIG, H. Isotopic variations in meteoric waters. Science, v. 133, p. 1702-1703. 1961.

DADRAS, Hadiseh et al. Effect of water temperature on the physiology of fish spermatozoon function: a brief review. Aquaculture Research, v. 48, n. 3, p. 729-740, 2017.

HOLMSTRUP, Martin et al. Interactions between effects of environmental chemicals and natural stressors: a review. Science of the Total Environment, v. 408, n. 18, p. 3746-3762, 2010.

HORNSTEIN, Jesse et al. The influence of temperature stress on the physiology of the Atlantic surfclam, Spisula solidissima. Comparative Biochemistry and Physiology Part A: Molecular \& Integrative Physiology, v. 222, p. 66-73, 2018. 